\title{
WHITE-LIGHT RECONSTRUCTION OF HOLOGRAPHIC IMAGES USING TRANSMISSION HOLOGRAMS RECORDED WITH CONVENTIONALLY- FOCUSED IMAGES AND 'IN-LINE' BACKGROUND
}

\author{
G. W. STROKE \\ The University of Michigan, Ann Arbor. Michigan
}

Received 21 September 1966

\begin{abstract}
A new extension of Gabor's wavefront-reconstruction principle permits to reconstruct three-dimensional images by transmission of white light through a hologram recorded in a new arrangement using anl 'illline' coherent background superposed onto a conventionally focused image field.
\end{abstract}

We have recently [1-3] been able to show (based in particular on electromagnetic field considerations; see also ref. 14) that it is possible to extend to 'focused' image regions the fundamental advantages introduced into optical imaging by Gabor's principle of wavefront-reconstruction imaging, which he originated in 1948 [4-6]. In particular, we have shown that it thus becomes possible to 'restore' the third-dimension information in the recording of conventionallyfocused photographs, when this may appear of advantage, for instance in certain telescopic and microscopic applications, among others. In these preliminary experiments $[1-3,7,11]$, the coherent background was introduced either 'offaxis' [8-10] or 'from the back of the cmulsion', in extension of our white-light Lippmann-Bragg reflection holography work, first described in ref. 11. The white-light holographic imaging method which we describe here differs in several ways quite fundamentally from our previously described white-light reflection holography work, of both the unfocused-beam $[11,12]$ and the focused-image [3] types. Our work with focusedimage holograms again indicates that Gabor's wavefront-reconstruction principle should be considered as being more fundamental than any of the particular arrangements used to record holograms capable of reconstructing threedimensional images.

The author has already previously proposed [10, page 121] in reference to transmission holograms, "that it should be possible to record phase information in a hologram wihtout Fresnel-zone "carrier fringes'" and that "in case of two waves having the same curvature and the same direction of propagation, the phase infor-

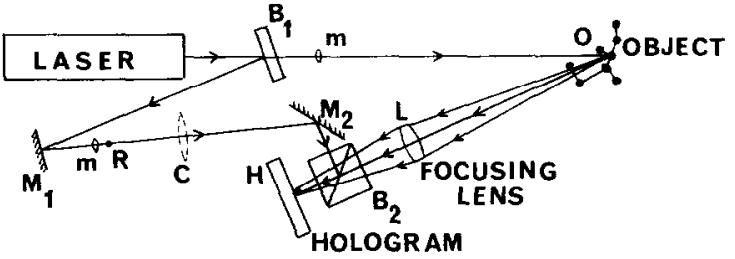

Fig. 1. Holographic recording arrangement used to record 'focused-image' holograms with 'in-line' background and capable of reconstructing three dimensional images by transmission of white light. $\mathrm{B}_{1}, \mathrm{~B}_{2}$ are beam-splitters, $\mathrm{M}=$ mirrors, $\mathrm{m}=$ microscope lenses, $\mathrm{C}=$ collimating lens (may be omitted with longradius beams), $\mathrm{L}=$ focusing lens used to produce a real image of the three-dimensional object $O$ near the hologram plate (for instance just in front of it), $\mathrm{R}=$ $=$ reference point (see text.)

mation is uniquely translated in the interferogram simply by the local intensity across the wavefront". Our present experiments with in line' reference beams superposed onto a 'focused' imaging field have now permitted to materialize conditions for this type of holographic recording. One suitable arrangerient is illustrated in fig. 1, in which a plane (or suitably spherical) coherent background wave was deliberately and carefully adjusted so as to be 'in-line' with the optical axis of the focusing lens $L$, used to form a real image of the three-dimensional object $O$ just in front of (or straddling) the hologram plate, with the optical axis approximately normal to the plate. (Details of the required optimum adjustment of the background-wave curvature relative to the image will be given in a subsequent paper [13]. Initial experiments indicate that the 'equicurvature' of the 'wavefronts' may be applied to the distance $\mathrm{OH}$ and $\mathrm{RM}_{2} \mathrm{H}$ of the object and the reference point $R$ to the hologram $H$, respectively) 

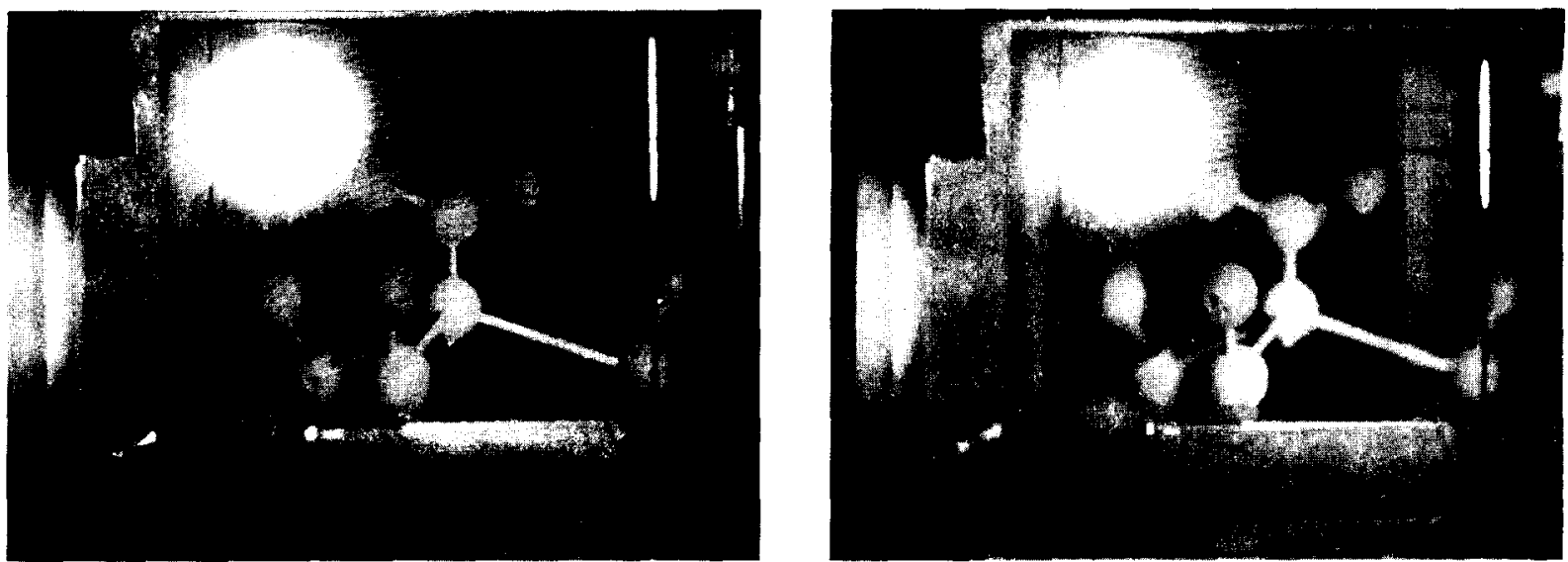

Fig.2. Two differently focused photographs of the three-dimensional image reconstructed from a hologram (recorded as in fig. 1) by transmission of white light frcm a carbon arc. Note depth of field, as illustrated by sharp focus on either the front or the rear portions of the reconstructed image. Note also clearly visible outline of hologram plate (including some writing) in fig. $2 a$, showing that the reconstructed image is reconstructed in the near vicinity of the hologram. (A lateral reduction of $\frac{1}{2}$, and axial reduction of about $\frac{1}{4}$ was used in the recording with an $18 \mathrm{~cm}$ focal length, $5 \mathrm{~cm}$ diameter lens, with the object of $15 \mathrm{~cm}$ deep, $10 \mathrm{~cm}$ high, $15 \mathrm{~cm}$ wide, 'imaged' on a $10 \times 12.5 \mathrm{~cm}$ photographic plate, with a $6328 \AA$ helium-neon laser). The carbon arc projection clearly appears in the upper left of both photographs.

The holograms obtained in the arrangement of fig. 1 are characterized by several remarkable properties, which differ sufficiently from previously described holograms, notably of the 'focusedimage' type $[1-3,7]$, to merit particular distinction. First, an excellent image is reconstructed (see fig. 2), even though difficulties might have been expected with the use of an 'in-line' reference background (e.g. those which tend to appear in comparable recordings with 'scattered beams', rather than with 'focused-images', as used here; residual image 'doubling' with the 'focusedimage' holograms is almost unnoticeable, except with deliberately imperfect alignment of the reference-beam direction). Second, the image can be readily reconstructed with this type of hologram by an 'in-line' transmission of a beam of ordinary white light (e.g. from an electric lamp, a carbon arc, the sun, etc.) without showing any spectral dispersion (sce also our observation in ref. 2). And finally, just like our previously described types of 'focused-image' holograms [1-3], the 'in-line' focused-image hologram also reconstructs a three-dimensional image, displaying parallax and depth of field, and the previously observed 'inversion' of the relief [2] (with the more distant parts of the object appearing closer to the observer in the reconstruction without any noticeable physiological annoyance); however, the reconstructed field is now wide (encompassing essentially the entire 'field' of the image which would be 'collapsed' onto the plate by the focusing lens in 'conventional' photography) regardless of which side of the hologram is illuminated in the reconstruction. (We may add that the images appear considerably brighter and even sharper than those which may be reconstructed from the 'white-light reflection holograms ' $[11,12]$ recorded on comparable emulsions). As an illustration of the three-dimensional nature of the reconstructed image, we have focused in fig. 2 the photographic camera (looking at the reconstructed image, formed typically in the vicinity of the hologram) respectively onto a front part and onto a rear part of the image, clearly indicating the depth of field in the reconstructed image.

The theory of the 'in-line focused-image holograms' may be readily given in simple terms. It may be sufficient to note that the recording of an 'in-line' hologram with a focused image field $\bar{E}_{\mathrm{O}}(x, y, z, t)$ and an 'in-line' reference field $\bar{E}_{\mathrm{R}}(x, y, z, t)$, both propagating in the same direction, results in the reconstruction with an 'inline' field $\bar{E}_{\mathrm{R}}$ (assume $\bar{E}_{\mathrm{R}}=1$ for simplicity) in a reconstructed image field equial to $\left[\left|\bar{E}_{\mathrm{O}}\right|^{2}+1\right]+$ $+\bar{E}_{\mathrm{O}}+\bar{E}_{\mathrm{O}}^{*}$, from which it may be readily seen that the two conjugate reconstructed waves $\bar{E}_{\mathrm{O}}$ and $\bar{E}_{\mathrm{o}}^{*}$ will be spatially superposed as observed. (For further clarifications, see also fig. 4 in ref. 15). The white-light reconstruction may be attributed to the absence of grating-like carrier fringes [10] (or, more generally, in the case of slight curvature differences between the wavefronts, to the very low 'spectral' dispersion associated with the very 'coarse' residual fringe 
spacing). Theoretical considerations (confirmed by the absence of satisfactory imaging with coarse emulsions) tend to indicate however, that the holographic information in these holograms is spread out in a 'correlated' way [14] over comparatively wide regions of the emulsion (centered as it were, on the geometrically focused or defocused image 'points'), as one would expect, as a result of the 'defocused' projection of the threedimensional images onto the plate. Accordingly, the use of the fine-grain normally -thick (17 micron) Kodak $649 \mathrm{~F}$ emulsions was found necessary in this work (taking into account also possibly helpful 'volume' effects).

The authors wishes to acknowledge fruitful private communications from and discussions with Professor Dennis Gabor, as well as his very kind interest and encouragement. He also wishes to acknowledge the kind assistance of his student R. G. Zech with the experimental work and many fruitful discussions with him. He also wishes to thank the National Science Foundation and the Office of Naval Research for support of this work.

1. L. Rosen, submitted to IEEE proc.

2. W.E.Kock, L. Rosen and G.W.Stroke, submitted to IEEE proc.

3. W. E. Kock, L.Rosen, G.W.Stroke, R.G. Zech and C. Leonard, Submitted to Phys. Rev. Letters.

4. D. Gabor, Nature 161 (1948) 777.

5. D. Gabor, Proc. Roy. Soc. (London) A197 (194) 454.

6. D. Gabor, Proc. Phys, Soc. (London) 64 (1951) 449.

7. R.F.Van Ligten and H. Osterberg, Nature 211 (1966) 282 .

8. L. J.Cutrona, E. N. Leith, C.J. Palermo and L.J. Porcello, IRF TMans. Inform. Theory, IT-6. (1960) 386.

9. E.N. Leith and J. Upatnieks, J. Opt. Soc. Am. 53 (1963) 981.

10. G.W. Stroke, An introduction to coherent optics and holography (Academic Press Inc., New York and London, 1966).

11. G.W.Stroke and A.E. Labeyrie, Physics Letters 20 (1966) 368.

12. L.H.Lin, K.S. Pennington, G.W. Stroke and A.E. Labeyrie, Bell. Syst. Tech. J. 54 (1966) 659.

13. G.W.Stroke and R. G.Zech, to be published.

14. G.W.Stroke, D.Brumm, A. Funkhouser, A. Labeyrie and R.C.Restrick, Brit. J. Appl. Phys. 17 (1966) 497.

\title{
PHASE TRANSITIONS IN SPIN-ONE ISING SYSTEMS
}

\author{
H. W. CAPEL \\ Institunt-Loventz voor Theoretische Natuurkunde, Leiden, Netherlands
}

Received 22 September 1966

\begin{abstract}
Two special $S=1$ (spin-one) Ising models can be related to the $S=\frac{1}{2}$ Ising model. Properties of the phase transitions are derived from the $S=3$ Ising model.
\end{abstract}

It has been shown in the molecular-field approximation that a $S=1$ Ising model defined by the Hamiltonian

$$
\mathscr{H}=-D \sum_{i}\left(1-S_{z i}^{2}\right)-J \sum_{\langle i, j\rangle} s_{z i} s_{z j}-\mu H \sum_{i} s_{z i}
$$

where $D$ is the zero-field splitting, $J$ the exchange interaction between pairs of nearest neighbours $\langle i, j\rangle, \mu$ the magnetic moment and $H$ an applied magnetic field, undergoes a firstorder transition under certain conditions [1,2]. A similar model has been used by Blume [3] in order to explain the first-order transition in
$\mathrm{UO}_{2}[4]$. In this paper we discuss two models which can be related to the $S=\frac{1}{2}$ Ising model.

In the limit $\mu H \rightarrow \infty, D \rightarrow \infty, \mu H-D=$ finite, the configurations with atoms $S_{Z}=-1$ do not contribute to the partition function of (1) and by substituting $\sigma_{i}=2 S_{z i}-1= \pm 1$ it can be shown that the free energy of the system apart from an additive constant is given by

$$
F=F_{\mathrm{I}}\left(H^{\prime}, J^{\prime}\right)
$$

where $F_{I}$ is the free energy of a $S=\frac{1}{2}$ Ising model with exchange interaction $J^{\prime}$ in a magnetic field $H^{\prime}$ with $\mu H^{\prime}=\frac{1}{2}\left\{\mu H-D+\frac{1}{2} z J\right\}$ and $J^{\prime}=\frac{1}{4} J$.

For positive values of $J$, there is one first- 